Article

\title{
Fiber Optic pH Sensor with Self-Assembled Polymer Multilayer Nanocoatings
}

\author{
Li-Yang Shao ${ }^{1,2, *}$, Ming-Jie Yin ${ }^{2}$, Hwa-Yaw Tam ${ }^{2}$ and Jacques Albert ${ }^{3}$ \\ 1 Institute of Optoelectronic Technology, China Jiliang University, Hangzhou 310018, China \\ 2 Department of Electrical Engineering, The Hong Kong Polytechnic University, \\ Hung Hom, Kowloon, Hong Kong; E-Mails: ming-jie.yin@ connect.polyu.hk (M.-J.Y.); \\ eehytam@polyu.edu.hk (H.-Y.T.) \\ 3 Department of Electronics, Carleton University, Ottawa, ON K1S 5B6, Canada; \\ E-Mail: Jacques_Albert@carleton.ca \\ * Author to whom correspondence should be addressed; E-Mail: eelyshao@ polyu.edu.hk; \\ Tel./Fax: +86-571-8767-6260.
}

Received: 7 December 2012; in revised form: 16 January 2013 / Accepted: 21 January 2013 / Published: 24 January 2013

\begin{abstract}
A fiber-optic pH sensor based on a tilted fiber Bragg grating (TFBG) with electrostatic self-assembly multilayer sensing film is presented. The $\mathrm{pH}$ sensitive polymeric film, poly(diallyldimethylammonium chloride) (PDDA) and poly(acrylic acid) (PAA) was deposited on the circumference of the TFBG with the layer-by-layer (LbL) electrostatic self-assembly technique. The PDDA/PAA film exhibits a reduction in refractive index by swelling in different $\mathrm{pH}$ solutions. This effect results in wavelength shifts and transmission changes in the spectrum of the TFBG. The peak amplitude of the dominant spectral fringes over a certain window of the transmission spectrum, obtained by FFT analysis, has a near-linear $\mathrm{pH}$ sensitivity of 117 arbitrary unit (a.u.)/pH unit and an accuracy of \pm 1 a.u. (in the range of $\mathrm{pH} 4.66$ to $\mathrm{pH}$ 6.02). The thickness and surface morphology of the sensing multilayer film were characterized to investigate their effects on the sensor's performance. The dynamic response of the sensor also has been studied (10 s rise time and $18 \mathrm{~s}$ fall time for a sensor with six bilayers of PDDA/PAA).
\end{abstract}

Keywords: fiber-optic sensor; layer-by-layer self-assembly; multilayer film; $\mathrm{pH}$ sensor; tilted fiber Bragg grating 


\section{Introduction}

$\mathrm{pH}$ monitoring is widely demanded in biomedical, environmental, and industrial fields such as clinical blood analysis, industrial wastewater treating, food processing, and process control in bioreactors [1]. However, the conventional $\mathrm{pH}$ electrodes are always too voluminous to be utilized for in vivo $\mathrm{pH}$ measurements, especially in medicine and clinical analysis [2]. Compared with electrical $\mathrm{pH}$ sensors, optical fiber-based $\mathrm{pH}$ sensors exhibit many advantages such as compact size, electrically passive operation, immunity to electromagnetic interference, capability of remote measurement and multiplexed detections [3,4]. Different fiber-optic $\mathrm{pH}$ sensors have been demonstrated by depositing a $\mathrm{pH}$-sensitive coating onto the fiber surface such as hydrogels [5], cellulosic films [6], sol-gel polymers [7]. Recently, layer-by-layer (LbL) electrostatic self-assembly (ESA) technique has emerged as a versatile and convenient method for preparing ultrathin multilayer films due to their precise control of thickness at the nanometer scale and deposition on non-flat surfaces [8-11]. Goicoechea demonstrated a Fabry-Perot (FP) nanocavity $\mathrm{pH}$ sensor by coating the nanostructured film on the fiber-end surface and white-light interferometry is employed for the measurement of $\mathrm{pH}$-induced swelling of the film [12]. Corres presented a long period grating (LPG) $\mathrm{pH}$ sensor where the nanocoating was deposited on the circumference of the LPG as the reacting functional film and the resonant wavelength shift of the LPG was utilized for the determination of $\mathrm{pH}$-induced refractive index (RI) changes [13]. Gu proposed a simple fiber-optic pH sensor based on the thin-core fiber modal interferometer (TCFMI) [2]. The LPG and TCFMI sensors have similar problems in locating the exact resonant wavelength because of their large bandwidths. The TCFMI scheme also has the disadvantage of high insertion loss due to the mismatch of fiber core diameter, which requires a relatively high power light source for reasonable signal to noise ratio and might limit its multiplexity ability.

In this paper, we propose a new fiber-optic $\mathrm{pH}$ sensor based on a TFBG with PDDA/PAA multilayer sensing films. The PDDA/PAA coating has a high refractive index (relative to the material of the fiber, i.e., silica), so not only the resonance wavelength but also the coupling strength change with the $\mathrm{pH}$ of the solution. We will show that the visibility of the transmission spectrum oscillations caused by cladding mode resonances increases with the $\mathrm{pH}$. Similar to our work on a TFBG pressure sensor [14], by applying a fast Fourier transform (FFT) analysis of a suitable region of the transmission spectrum, we obtain a direct measure of the visibility and hence of the $\mathrm{pH}$ of the solution. Another advantage of the TFBG pH sensor is its inherent temperature reference that is provided by the core mode reflection wavelength. Finally, dynamic measurements show that fast responses were obtained with a rise time $\left(t_{r}\right)$ of $10 \mathrm{~s}$ and fall time $\left(t_{\mathrm{f}}\right)$ of $18 \mathrm{~s}$ for the sensor with six bilayers of PDDA/PAA.

\section{Experimental Procedures}

\subsection{Materials}

Poly(diallyldimethylammonium chloride) (PDDA, $\mathrm{Mw}=100,000 \sim 200,000 \mathrm{~g} \cdot \mathrm{mol}^{-1}, 20 \%$ aqueous solution, refractive index: 1.375 ), and poly(acrylic acid) (PAA, $\mathrm{Mw}=100,000 \mathrm{~g} \cdot \mathrm{mol}^{-1}, 35 \%$ aqueous solution, refractive index: 1.393) were purchased from Aldrich (St. Louis, MO, USA). Hydrochloric acid $(\mathrm{HCl})$ and sodium hydroxide $(\mathrm{NaOH})$ were all analytical reagents. Deionized water with a resistance of $18 \mathrm{M} \Omega \mathrm{cm}$ was used in all experiments. 


\subsection{Preparation of (PDDA/PAA $)_{n}$ Multilayer Films}

The preparation process is similar with our previous works $[3,15,16]$. Both PDDA and PAA solutions were prepared with a concentration of $2.0 \mathrm{~g} \cdot \mathrm{L}^{-1}$ and a $\mathrm{pH}$ of 3.0. The $\mathrm{pH}$ of all solutions was checked with a digital precision pH meter (model PHS-5E, Changhong Ke Ji, Shenzhen, China), with an accuracy of $\pm 0.01 \mathrm{pH}$. The LbL electrostatic self-assembly of oppositely charged polyelectrolytes (positively charged PDDA and negatively charged PAA) was carried out on the substrates (here they were AT-cut quartz crystals with gold electrode, quartz slides $\left(10 \times 20 \mathrm{~mm}^{2}\right)$ and TFBG. The substrates were cleaned by piranha solution $\left(8: 2\right.$ of $95 \% \mathrm{H}_{2} \mathrm{SO}_{4}$ and $50 \% \mathrm{H}_{2} \mathrm{O}_{2}$ ), washed with large amounts of deionized water and dried with nitrogen, obtaining negatively charged substrates. The negatively charged substrates were dipped into the positively charged PDDA and negatively charged PAA solutions alternatively, each for $10 \mathrm{~min}$ at room temperature. Between polycation and polyanion immersions, the substrates were rinsed in deionized water three times ( 1 min each time) to remove any excess of adsorbed material and dried with nitrogen. A coating made by two dipping procedures was called a bilayer, named as (PDDA/PAA $)_{1}$. The same cycle was repeated until the desired number of bilayers was deposited.

\subsection{Thin Film Characterizations}

The fabrication process and thickness of the multilayer film were traced by quartz crystal microbalance measurements (QCM, Resonance Probe GmbH, Goslar, Germany). AT-cut quartz crystals (Maxtek, Shanghai, China) with a fundamental frequency $f_{0}$ of $5 \mathrm{MHz}$ and gold electrodes were used. The multilayer films were fabricated on the front side of the quartz crystal. The frequency shift of the quartz crystal, $\Delta f$, after the deposition of each layer was measured at three overtone orders $\mathrm{n}=1,3,5$ (i.e. 5,15 , and $25 \mathrm{MHz}$ ) and the corresponding thickness was estimated with the Sauerbrey equation with the assumption that the density of the multilayer film is $1.0 \mathrm{~g} \cdot \mathrm{cm}^{-3}$ [15] in Equation (1):

$$
d_{f}=-\frac{Z_{q} \cdot \Delta f}{2 f_{0} \cdot f \cdot \rho_{f}}=\left(-5.87 \times 10^{-2}\right) \Delta f(\mathrm{~nm})
$$

where $\Delta f$ is frequency shift, $f=\mathrm{n} f_{0}$ is the frequency, $\rho_{f}$ is the density of the film, and $Z_{q}=8.8 \times 10^{6} \mathrm{~kg} \cdot \mathrm{m}^{-2} \cdot \mathrm{s}^{-1}$ is the acoustic impedance of crystalline quartz.

Atomic force microscopy measurements (AFM, tapping mode) were performed by using a Seiko SPI3800N station (Seiko Instruments Inc., Tokyo, Japan). Silicon tips (NSG10, NT-MDT) with a resonance frequency of $c a .330 \mathrm{kHz}$ were used.

\subsection{Grating Fabrication}

Corning SMF-28 fiber was used to fabricate the TFBGs. The fiber was soaked in a hydrogen chamber for 12 days at $2,500 \mathrm{psi}$ and room temperature of $25^{\circ} \mathrm{C}$ to improve its photosensitivity. 1-cm-long TFBG with a tilt angle of $10^{\circ}$ was inscribed in the hydrogen-loaded fibers using a pulsed $\mathrm{KrF}$ excimer laser and the phase mask technique. To excite high order cladding modes spectrally located in the $\mathrm{C}+\mathrm{L}$ band $(1,520-1,610 \mathrm{~nm})$, the period of phase mask was chosen to be $1,106.5 \mathrm{~nm}$. The resulting Bragg wavelength of TFBG is 1,602.36 nm. A broadband light source and optical 
spectrum analyzer were used to measure the spectrum evolution of the sensing TFBG. Another optical spectrum analyzer with sampling rate of $1 \mathrm{kHz}$ and bandwidth from 1,510 nm to 1,590 nm (from Micron Optics, Inc., Atlanta, GA, USA) was utilized for the dynamic measurement.

\section{Results and Discussion}

The PDDA/PAA multilayer films were firstly deposited on AT-cut quartz crystals to study the LbL assembly process using QCM. Figure 1 shows that the thickness of multilayer films grows almost linearly with increasing layer numbers. this indicates uniform film stacking by the LbL method [3,17]. The thickness of (PDDA/PAA) 10 multilayer film is about $185 \mathrm{~nm}$, which means about $9 \mathrm{~nm}$ for each layer thickness. Because of this stackability, the thickness of the multilayer films fabricated by LbL can be controlled to within a few nanometers. This control is very important for preparing fiber-optic sensors, as some types of sensors have a very short range of penetration of the optical probing field outside of the fiber [4].

Figure 1. Thickness measurement with different number of bilayers. The insert shows the schematic diagram of LbL electrostatic self-assembly multilayers.

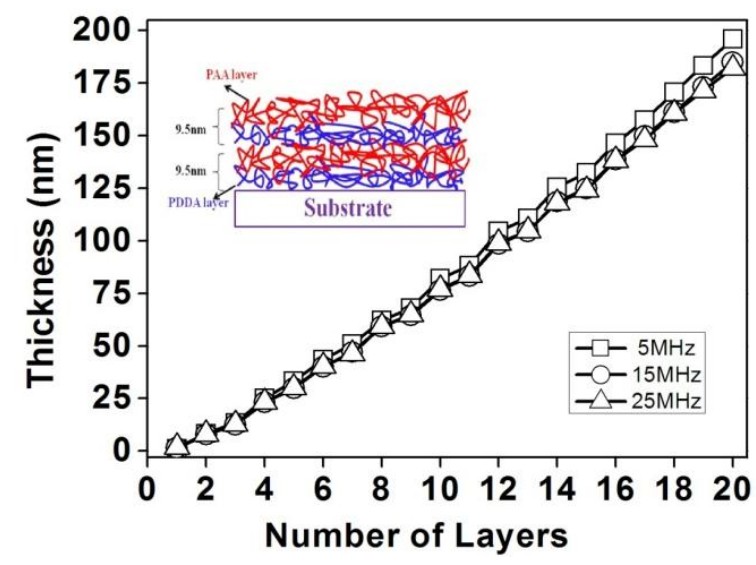

Figure 2. AFM images of multilayer nanocoatings (a) $(\mathrm{PDDA} / \mathrm{PAA})_{4}$ and $(\mathbf{b})(\mathrm{PDDA} / \mathrm{PAA})_{10}$.
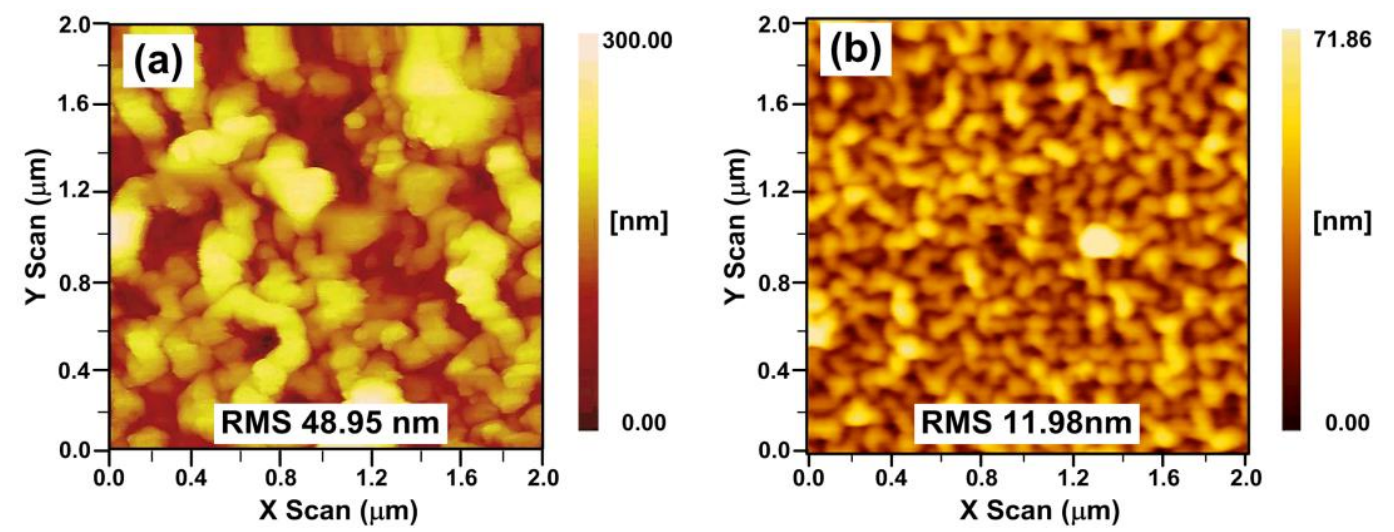

Figure 2 gives the atomic force microscopy (AFM) images of (PDDA/PAA) 4 and (PDDA/PAA) 10 multilayer films (dry). A wormlike or vermiculate pattern can be observed for the multilayer, which is a general phenomenon for common polyelectrolyte multilayer films [3,18]. However, comparing Figure $2(a, b)$, the morphology of the (PDDA/PAA $)_{4}$ multilayer film has smaller, less connected 
aggregates and larger root mean square (RMS) height, that is $48.95 \mathrm{~nm}$, while for the (PDDA/PAA) 10 multilayer film, larger clusters are formed that tend to connect together, with a smaller RMS of $11.98 \mathrm{~nm}$. The reason is that as the layer number increases, the polyelectrolyte chains will fill up the surface voids of previous multilayer film [19].

We also tested the water contact angles (CA) for PDDA/PAA multilayer films. Figure 3 shows how the CA changes with the number of layers. It can be seen that the CA for PDDA as the outermost layer is about $30^{\circ}$ while with PAA as the outermost layer it is about $25^{\circ}$, which means a strong hydrophilic property of the sensing film, so the PDDA/PAA multilayer films are suitable for $\mathrm{pH}$ sensing as the test is in water solutions.

Figure 3. Water contact angles (CA) for PDDA/PAA multilayer films.

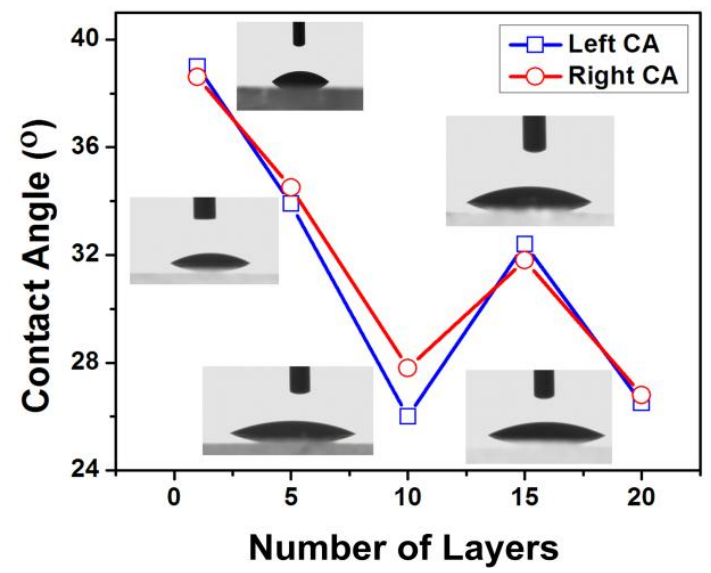

Figure 4 shows the transmission spectra of a $10^{\circ}$ TFBG without coating and with 4,6 and 10 bilayers of PDDA/PAA multilayer sensing film. When the sensing film gets thicker, the high-order cladding mode resonances are largely suppressed and a broadband radiation loss exists due to the coupling from the core mode to the radiation mode continuum. That is because when the RI of surrounding medium increases to or over that of the fiber cladding, the coupling strength of higher-order cladding mode decreases strongly. Figure 4 also further demonstrates that our PDDA/PAA multilayer sensing film have been successfully deposited on the surface of the TFBG.

Figure 4. Transmission spectra of $10^{\circ}$ TFBG without coating and with 4, 6 and 10 bilayers $\left[(\mathrm{PDDA} / \mathrm{PAA})_{4},(\mathrm{PDDA} / \mathrm{PAA})_{6},(\mathrm{PDDA} / \mathrm{PAA})_{10}\right]$.

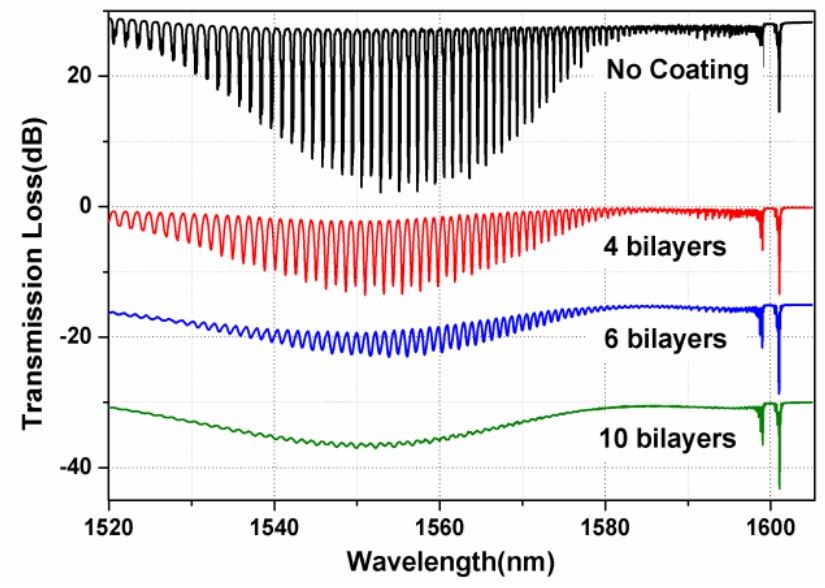


The fabricated TFBG pH sensor was packaged into a shallow alumimum box with two ends of grating fixed to avoid the bend of fiber affecting on the results of $\mathrm{pH}$ measurement. Three sensors with $(\mathrm{PDDA} / \mathrm{PAA})_{4},(\mathrm{PDDA} / \mathrm{PAA})_{6},(\mathrm{PDDA} / \mathrm{PAA})_{10}$ multilayer sensing film have been packaged and tested one by one. The principle of our TFBG based $\mathrm{pH}$ sensor is shown in Figure 5. As the swelling state of the PDDA/PAA multilayer sensing film changes with the $\mathrm{pH}$ of the solution, the RI of the sensing film will also change accordingly [11,20]. As a result, the transmission spectra of the fiber-optic sensors will also be impacted.

Figure 5. Schematic principle of TFBG based pH sensor.

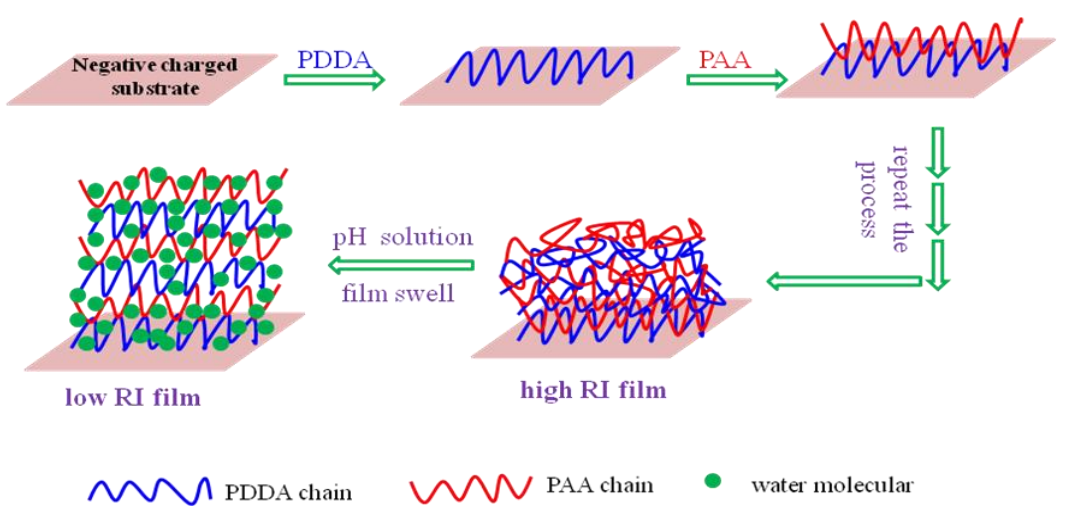

Figure 6. Evolution of optical spectra of TFBG $\mathrm{pH}$ sensors with different coatings $\left[(\mathbf{a})(\mathrm{PDDA} / \mathrm{PAA})_{4},(\mathbf{b})(\mathrm{PDDA} / \mathrm{PAA})_{6},(\mathbf{c})(\mathrm{PDDA} / \mathrm{PAA})_{10}\right]$.
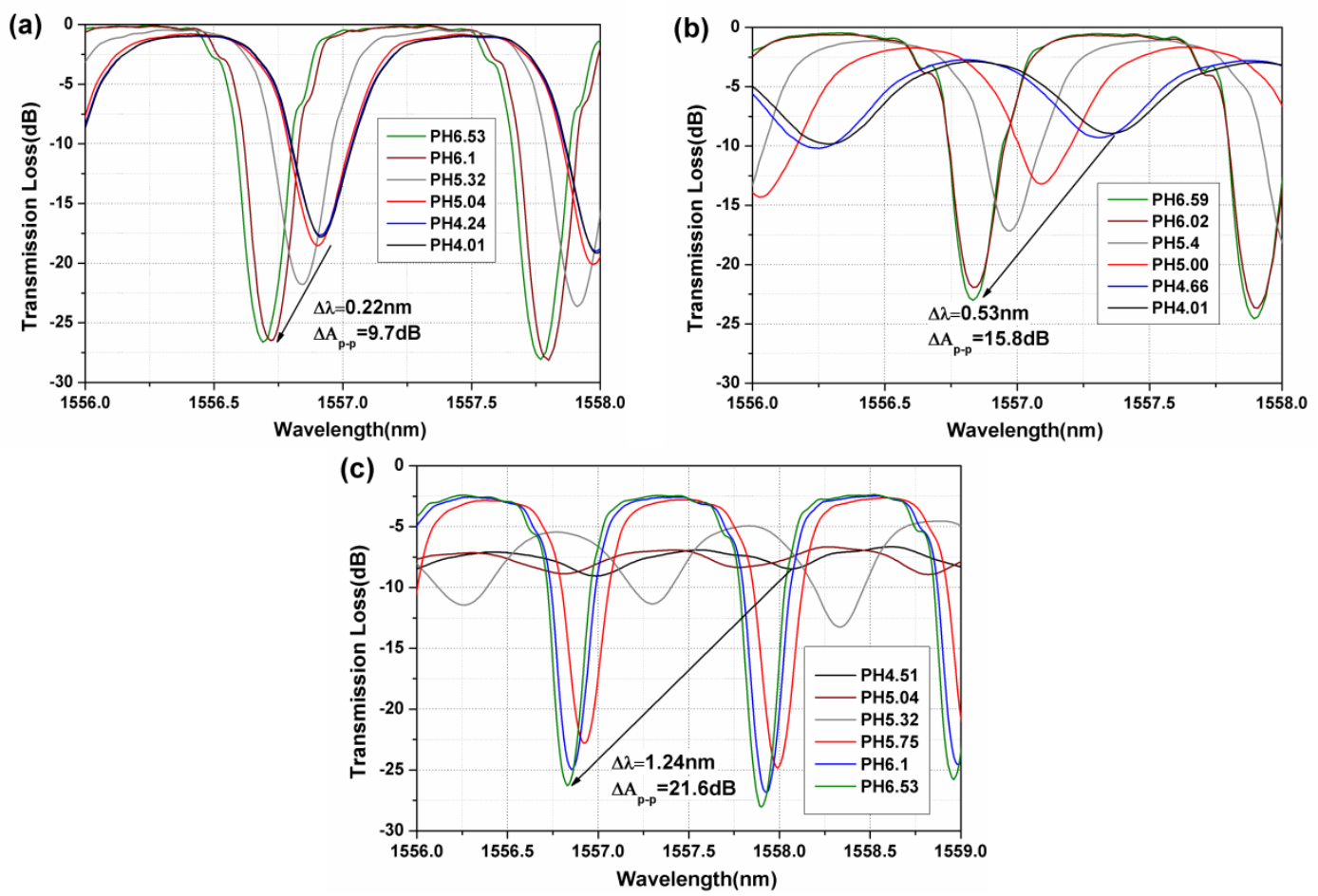

Figure 6 gives the spectral evolution of our fiber-optic sensors with $\mathrm{pH}$ for different thicknesses of sensing film. For each film, the $\mathrm{pH}$ of the aqueous solutions was adjusted by $\mathrm{HCl}$ or $\mathrm{NaOH}$ solution (in the range from 3.4 to 7.24). It can be seen that the $\mathrm{pH}$ increases, the coupling strength of the cladding mode resonances also increases. The reason is that with increasing $\mathrm{pH}$, more carboxyl groups 
will be ionized and the sensing film becomes more hydrophilic. This in turn leads to swelling of the sensing film to allow more water inside it, and reduces the RI [3]. Due to the effective RI decrease of the sensing film the cladding modes become more strongly guided and less lossy, thereby recuperating some cumulative coupling strength along the grating As a result, the visibility of the fringe pattern observed in the transmission spectrum increases with the $\mathrm{pH}$, a feature that will be used for quantitative analysis in a later section.

Figure 7 shows the wavelength shift and amplitude changes of the resonance around 1,557 nm. For the sensors with 4, 6 and 10 bilayers of (PDDA/PAA) film, the wavelength shifts are $0.22,0.53$ and $1.24 \mathrm{~nm}$, while the relative amplitude change is $9.7,15.8$ and $21.6 \mathrm{~dB}$ when the $\mathrm{pH}$ increases from 4.01 to 6.59. It is further observed that for certain ranges of the thickness of sensing film, the sensitivity (the slope of the curves in the Figures) also becomes larger.

Figure 7. (a) Wavelength shift and (b) amplitude change of one resonance around $1,557 \mathrm{~nm}$ for three sensors with coatings having different numbers of bilayers $(4,6,10)$.
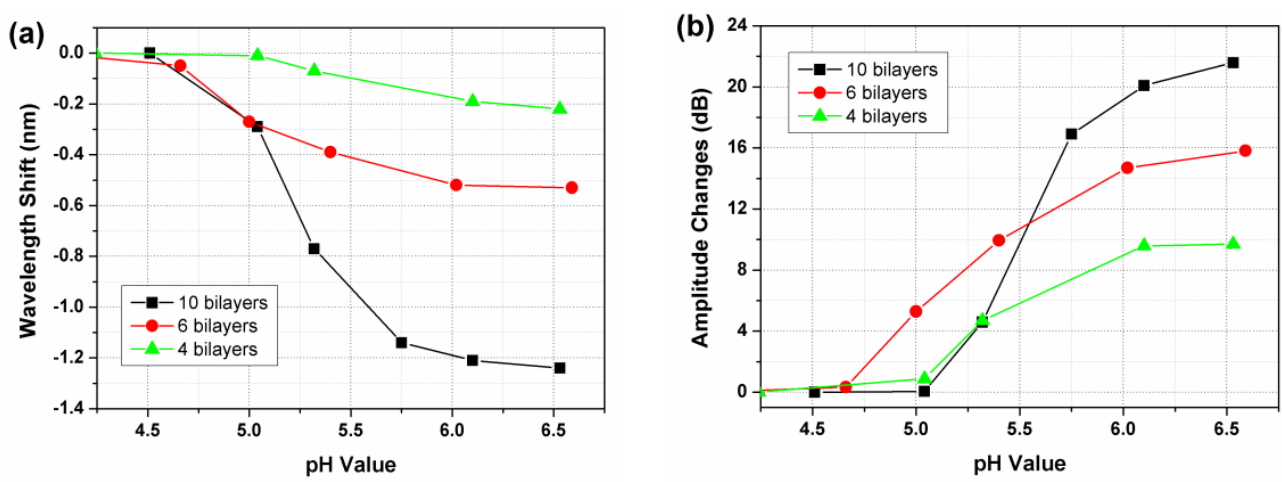

For the three fiber-optic $\mathrm{pH}$ sensors that we prepared, the (PDDA/PAA) ${ }_{10}$ multilayer sensing film gives the highest overall sensitivity while the (PDDA/PAA) $)_{4}$ multilayer has the lowest one. This makes sense as the thicker initial coating has the highest effective RI, as felt by the evanescent field of the cladding mode, and hence the highest reaction to the uptake of water (with low RI) that comes about with the swelling [21]. We found the sensitivity of the sensor with (PDDA/PAA) 4 to be too low because of the thinness of the sensitive coating, while the working range (linear response region) of the sensor with (PDDA/PAA) 10 is too narrow.

The dynamic response of the $\mathrm{pH}$ sensor was also measured in the experiment. The MOI optical spectrum analyzer keeps sweeping with a sampling rate of $1 \mathrm{~Hz}$. The response time (rise time) for the three fiber-optic sensors are 5, 10 and $30 \mathrm{~s}$, respectively. It can be found that as the number of bilayers increases the response time becomes longer. Two reasons account for that. The longer diffusion time for water molecules from the outside of the film towards the fiber surface, and the increased density of thicker films (less porous as seen on Figure 2), which decreases the diffusion coefficient itself. For the films that we used, the best compromise for the time response and $\mathrm{pH}$ sensitivity, is the TFBG $\mathrm{pH}$ sensor with the (PDDA/PAA) 6 coating.

Since both wavelength shifts measurements and the determination of the "amplitude" of the oscillations in the spectrum are somewhat arbitrary, we now propose a non-equivocal quantitative measure of the effect of changing $\mathrm{pH}$ on the sensor response. To emphasize the visibility response of 
the sensor with (PDDA/PAA) 6 coating, a band from $1,520 \mathrm{~nm}$ to $1,585 \mathrm{~nm}$ was selected for FFT analysis. Figure 8(a) depicts the results of this FFT analysis (using a Hanning window), while Figure 8(b) shows the amplitude of the dominant frequency peak around $0.8 \mathrm{~nm}^{-1}$ as a function of $\mathrm{pH}$. One can see the response is linear for $\mathrm{pH}$ values in the range of 4.66 to 6.02 . The obtained sensitivity is $117 \pm 1$ a.u./pH unit, and with an accuracy of $0.01 \mathrm{pH}$. The response out of this range is anomalous, because from the low $\mathrm{pH}$ side, the effective RI of sensing polymer coating is close to that of water, while the high $\mathrm{pH}$ side, the effective RI is comparable to that of the fiber cladding. Both of two cases are out of the dynamic RI sensing range of TFBG itself (from 1.33 to 1.44 RIU) [22].

Figure 8. (a) Corresponding FFT spectra of TFBG pH sensor with (PDDA/PAA) 6 coating. (b) The peak amplitude around $0.8 \mathrm{~nm}^{-1}$ changes with the $\mathrm{pH}$ of the solutions.
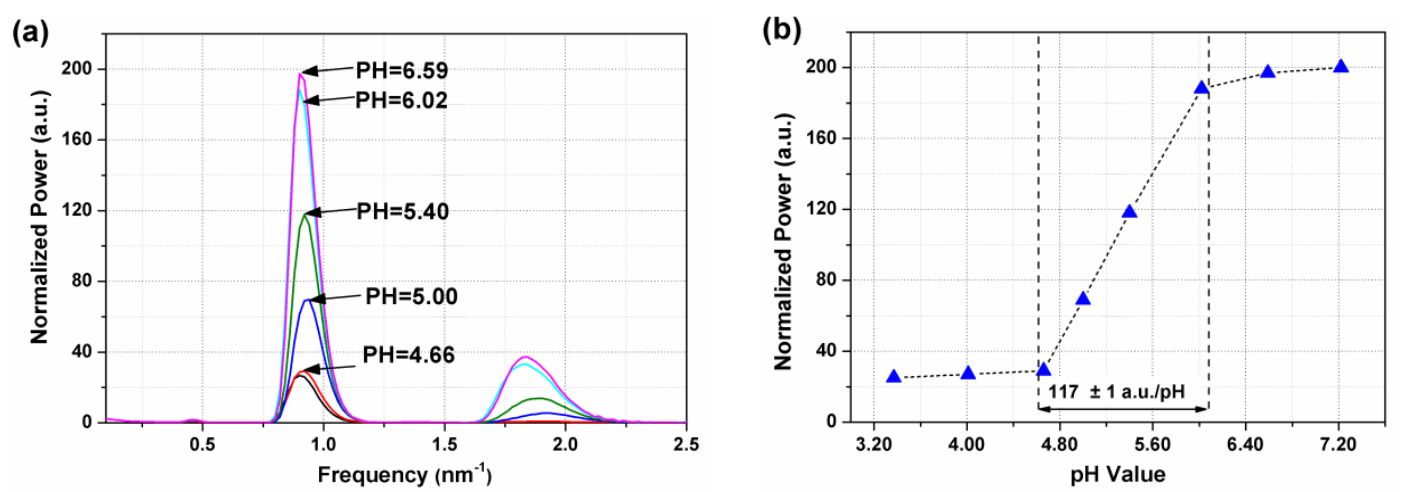

For the dynamic response measurement, buffer solutions with $\mathrm{pH}$ values of 4.66 and 6.02 are alternately added into the testing groove. Figure 9 illustrates the measured dynamic response of the TFBG $\mathrm{pH}$ sensor with the (PDDA/PAA) 6 coating, where the rise time $\left(\mathrm{t}_{\mathrm{r}}\right)$ of the sensor is $10 \mathrm{~s}$ and the fall time $\left(t_{f}\right)$ is $18 \mathrm{~s}$. The response time for all our TFBG based sensors is shorter than the results of previous works [2,3,21,23]. As the AFM-measured surface morphology shows in Figure 2, these nanocoatings have high RMS so that they absorbs/desorbs the water efficiently and fast. Consequently, the swelling/shrinking process of the nanocoating exposed to solutions with different $\mathrm{pH}$ can be more efficient.

Figure 9. The measured dynamic response of the TFBG pH sensor with (PDDA/PAA) 6 .

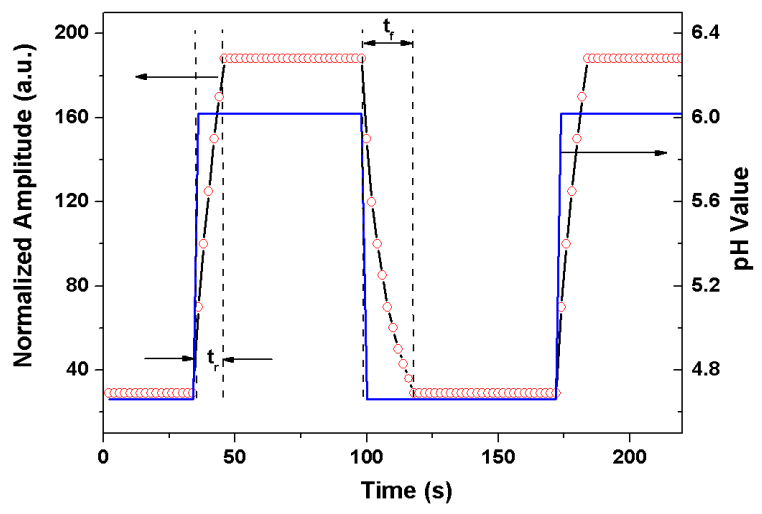




\section{Conclusions}

A polyelectrolyte sensing film has been successfully deposited on TFBG surfaces by the LbL electrostatic self-assembly technique to fabricate a novel fiber-optic $\mathrm{pH}$ sensor. The nanocoating was characterized by QCM and AFM. The main benefit of this kind of coating is its high RMS porosity, which makes the dynamic response very fast, of the order of 10 seconds. Our quantitative analysis technique for the sensor response yields a $\mathrm{pH}$ accuracy of 0.01 , sufficient for many biomedical and process monitoring applications, especially given the fact that this miniature sensor (less than $1 \mathrm{~cm}$ long, with a 125 micrometer diameter) can easily be interrogated remotely. A disadvantage of this sensor is the limited linear operating range of $\mathrm{pH}$ (from 4.66 to 6.02) due to the high RI of the coating. We expect that other polyelectrolytes or hydrogels with lower RI can be mixed into the coating to reduce the effective RI and increase the measurement range. Thickness optimization is also in progress to obtain higher $\mathrm{pH}$ sensitivity.

\section{Acknowledgments}

This work is supported by the Hong Kong Polytechnic University project G-YX5C, the Canada Research Chairs program and the Natural Sciences and Engineering Research Council of Canada (J. Albert), and the National Natural Science Foundation of China under Grant No. 61007050.

\section{References}

1. Lemos, S.G.; Nogueira, A.R.A.; Torre-Neto, A.; Parra, A.; Alonso, J. Soil calcium and pH monitoring sensor system. J. Agric. Food Chem. 2007, 55, 4658-4663.

2. Gu, B.; Yin, M.-J.; Zhang, A.P.; Qian, J.-W.; He, S. Low-cost high-performance fiber-optic pH sensor based on thin-core fiber modal interferometer. Opt. Express 2009, 17, 22296-22302.

3. Yin, M.; Gu, B.; Zhao, Q.; Qian, J.; Zhang, A.; An, Q.; He, S. Highly sensitive and fast responsive fiber-optic modal interferometric $\mathrm{pH}$ sensor based on polyelectrolyte complex and polyelectrolyte self-assembled nanocoating. Anal. Bioanal. Chem. 2011, 399, 3623-3631.

4. Yin, M.; Gu, B.; Qian, J.; Zhang, A.P.; An, Q.; He, S. Highly sensitive and selective fiber-optic modal interferometric sensor for detecting trace mercury ion in aqueous solution. Anal. Methods 2012, 4, 1292-1297.

5. Michie, W.C.; Culshaw, B.; Konstantaki, M.; McKenzie, I.; Kelly, S.; Graham, N.B.; Moran, C. Distributed $\mathrm{pH}$ and water detection using fiber-optic sensors and hydrogels. J. Lightwave Technol. 1995, 13, 1415-1420.

6. Jones, T.P.; Porter, M.D. Optical pH sensor based on the chemical modification of a porous polymer film. Anal. Chem. 1988, 60, 404-406.

7. Wolfbeis, O.S.; Offenbacher, H. Fluorescence sensor for monitoring ionic strength and physiological pH values. Sens. Actuators B 1986, 9, 85-91.

8. Hammond, P.T. Form and function in multilayer assembly: New applications at the nanoscale. Adv. Mater. 2004, 16, 1271-1293.

9. Decher, G. Fuzzy nanoassemblies: Toward layered polymeric multicomposites. Science 1997, 277, 1232-1237. 
10. Yin, M.; Qian, J.; An, Q.; Zhao, Q.; Gui, Z.; Li, J. Polyelectrolyte layer-by-layer self-assembly at vibration condition and the pervaporation performance of assembly multilayer films in dehydration of isopropanol. J. Membr. Sci. 2010, 358, 43-50.

11. Itano, K.; Choi, J.Y.; Rubner, M.F. Mechanism of the pH-induced discontinuous swelling/deswelling transitions of poly(allylamine hydrochloride)-containing polyelectrolyte multilayer films. Macromolecules 2005, 38, 3450-3460.

12. Goicoechea, J.; Zamarreño, C.R.; Matias, I.R.; Arregui, F.J. Utilization of white light interferometry in $\mathrm{pH}$ sensing applications by mean of the fabrication of nanostructured cavities. Sens. Actuators B 2009, 138, 613-618.

13. Corres, J.M.; Villar, D.I.; Matias, I.R.; Arregui, F.J. Fiber-optic pH-sensors in long-period fiber gratings using electrostatic self-assembly. Opt. Lett. 2007, 32, 29-31.

14. Shao, L.-Y.; Jiang, Q.; Albert, J. Fiber optic pressure sensing with conforming elastomers. Appl. Opt. 2010, 49, 6784-6788.

15. Zhang, P.; Qian, J.; An, Q.; Du, B.; Liu, X.; Zhao, Q. Influences of solution property and charge density on the self-assembly behavior of water-insoluble polyelectrolyte sulfonated poly(sulphone) sodium salts. Langmuir 2008, 24, 2110-2117.

16. Zhao, Q.; Qian, J.; An, Q.; Gui, Z.; Jin, H.; Yin, M. Pervaporation dehydration of isopropanol using homogeneous polyelectrolyte complex membranes of poly(diallyldimethylammonium chloride)/sodium carboxymethyl cellulose. J. Membr. Sci. 2009, 329, 175-182.

17. Wang, L.Y.; Wang, Z.Q.; Zhang, X.; Shen, J.C.; Chi, L.F.; Fuchs, H. A new approach for the fabrication of an alternating multilayer film of poly(4-vinylpyridine) and poly(acrylic acid) based on hydrogen bonding. Macromol. Rapid Commun. 1997, 18, 509-514.

18. McAloney, R.A.; Sinyor, M.; Dudnik, V.; Goh, M.C. Atomic force microscopy studies of salt effects on polyelectrolyte multilayer film morphology. Langmuir 2001, 17, 6655-6663.

19. Yang, Y.-H.; Haile, M.; Park, Y.T.; Malek, F.A.; Grunlan, J.C. Super gas barrier of all-polymer multilayer thin films. Macromolecules 2011, 44, 1450-1459.

20. Burke, S.E.; Barrett, C.J. pH-dependent loading and release behavior of small hydrophilic molecules in weak polyelectrolyte multilayer films. Macromolecules 2004, 37, 5375-5384.

21. Jesus, M.C.; Ignacio, R.M.; Ignacio, D.V.; Francisco, J.A. Design of pH sensors in long-period fiber gratings using polymeric nanocoatings. Sens. J. 2007, 7, 455-463.

22. Miao, Y.-P.; Liu, B.; Zhao, Q.-D. Refractive index sensor based on measuring the transmission power of tilted fiber Bragg grating. Opt. Fiber Technol. 2009, 15, 233-236.

23. Zamarreño, C.R.; Bravo, J.; Goicoechea, J.; Matias, I.R.; Arregui, F.J. Response time enhancement of $\mathrm{pH}$ sensing films by means of hydrophilic nanostructured coatings. Sens. Actuators B 2007, 128, 138-144.

(C) 2013 by the authors; licensee MDPI, Basel, Switzerland. This article is an open access article distributed under the terms and conditions of the Creative Commons Attribution license (http://creativecommons.org/licenses/by/3.0/). 